Review Articles

\title{
A Review on Challenging Issues in Arabic Sentiment Analysis
}

\author{
${ }^{1}$ Ali Hamdi, ${ }^{2}$ Khaled Shaban and ${ }^{1}$ Anazida Zainal \\ ${ }^{I}$ Faculty of Computer Science and Information Systems, Universiti Teknologi Malaysia, Johor, Malaysia \\ ${ }^{2}$ Department of Computer Science and Engineering, College of Engineering, Qatar University, Doha, Qatar
}

Article history

Received: 07-09-2016

Revised: 11-11-2016

Accepted: 10-12-2016

Corresponding Author:

Ali Hamdi

Faculty of Computer Science and Information Systems,

Universiti Teknologi Malaysia,

Johor, Malaysia

Email: ali@alihamdi.com

\begin{abstract}
Understanding what people think about an idea or how they evaluate a product, a service or a policy is important for individuals, companies and governments. Sentiment analysis is the process of automatically identifying opinions expressed in text on certain subjects. The accuracy of sentiment analysis has a direct effect on decision making in both business and government. Working with the Arabic language is very important because of the growing number of online contents in Arabic and the existing resources are limited and the accuracy of existing methods is low. In this study, we do a survey to highlight Arabic sentiment analysis challenging issues based on two main perspectives: Arabic-specific and general linguistic issues. The Arabic-specific challenges are mainly caused by Arabic morphological complexity, limited resources and dialects, while the general linguistic issues include polarity fuzziness, polarity strength, implicit sentiment, sarcasm, spam, review quality and domain dependence.
\end{abstract}

Keywords: Sentiment Analysis, Opinion Mining, Computational Linguistics, Text Classification

\section{Introduction}

The use of microblogging services has led to wide spread availability of opinionated posts (El-Beltagy and Ali, 2013). These available data provide an advantage for using social media websites and blogs in opinion studies. Understanding what people think about an idea or how they evaluate a product, a service or a policy is important for individuals, companies and governments. Sentiment Analysis (SA), also referred to in the literature as opinion mining, is the process of automatically identifying opinions expressed in text on certain subjects (Baly et al., 2016). SA has been performed on various levels of granularity; word, phrase, sentence (Wiebe et al., 1999), document (Pang et al., 2002; Turney, 2002), or aspect (Pontiki et al., 2014; 2015; Negi and Buitelaar, 2014) and from different perspectives; subjectivity identification or sentiment analysis. In order to apply SA, two main approaches are adopted: Lexicon-based and machine-learning approaches. Lexicon-based approaches use a dictionary of subjective words with their polarities and use simple matching methods to calculate the polarity scores (Al-Kabi et al., 2013; Li and Li, 2013; Badaro et al., 2014a). Machine-learning approaches use annotated datasets to train classifiers such as Support Vector Machines (SVM) (Kontopoulos et al., 2013; Tang et al., 2014), Naïve Bayes (NB) (Alhumoud et al., 2015; Farra et al., 2010), Neural Networks (NN) (Sharma and
Dey, 2012; Bollen et al., 2011) and more recently Deep Learning NN (Socher et al., 2013; Yuan and Zhou, 2015; Al Sallab et al., 2015). However, the obtained results are generally low in terms of accuracy especially in languages other than English such as the Arabic language on which we focus in this study.

According to the Internet World State rank in June 2016, Arabic is the fourth of the top ten languages used in the Internet (IWS, 2016). Most efforts in SA are focused on English and other Indo-European languages and little work has been done on Arabic (El-Halees, 2011; Abdul-Mageed et al., 2011). Most of the SA methods have been developed for English text and are difficult to apply to other languages like Arabic (Al-Kabi et al., 2014). Arabic is a morphologically rich language that poses significant challenges to Natural Language Processing (NLP) systems in general (Abdul-Mageed et al., 2011). Annotated Arabic corpora, necessary for training machine learning classifiers, are not only small, but also rare to be publically available. Moreover, almost all efforts on Arabic SA are focused on processing text in the general domain or in text from news articles and little is developed for targeted and specific domains such as finance, sports, legal, etc. Assiri et al. (2015) reported that this lack of support for the Arabic language is due to the limited scholarly work and research fund and the morphological complexities and different dialects of the Arabic language. 
There are many survey studies covering SA. For example, alOwisheq et al. (2016) reviewed works pertaining to the recent resources (i.e., lexica and corpora) which have been targeting the Arabic language, Korayem (2016) studied the sentiment and subjectivity methods for languages other than English, Schouten and Frasincar (2015) focused on the aspect detection for SA, Korayem et al. (2012) surveyed different techniques for subjectivity and SA of the Arabic language, Vinodhini and Chandrasekaran (2014) discussed the different SA techniques, methods and applications and other survey papers like (Yadav, 2015; Sadegh et al., 2012; Buche et al., 2013; Mahadik and Bharambe, 2015; Wiegand et al., 2010; Tang et al., 2009; Kaur and Duhan, 2015; Ahire, 2014; Liu and Zhang, 2012; Assiri et al., 2015). Moreover, El-Beltagy and Ali (2013) discussed some of the SA open issues in Arabic social media. These previous surveys focused on recent works categorization, SA techniques and applications. While some of them highlight the SA challenging issues, differently and albeit more comprehensively, the current manuscript attempts to cover such issues, discuss their causes to the SA low accuracy problem, focus on the Arabic language and highlight how previous work dealt with those issues.

\section{Arabic Sentiment Analysis Issues}

The main aim in any SA work is to produce highly accurate results, thus we discuss the challenges which contribute to low accuracy in Arabic SA. Figure 1 illustrates the challenging issues, where these issues are divided based on two main perspectives; Arabic-specific and general linguistic issues that are common to all languages.

\section{Arabic-Specific Challenges}

Arabic, the language considered in this study, introduces additional difficulties when developing SA systems because of its morphological complexity, the existence of a large number of dialectal variants and the lack of resources. The Arabic language has a complex morphological structure based on root-pattern schemes (Al-Sughaiyer and Al-Kharashi, 2004). Also it has many variants, such as classical Arabic, which is the language of the Quran; modern standard Arabic (MSA), the official language that is standardized, written in news and taught in schools; and dialectal Arabic (DA), which is used in daily lives and spoken communications (Zaidan and Callison-Burch, 2011; Habash, 2010). Arabic used in social media is usually a mixture of MSA and one or more of the Arabic dialects (Refaee and Rieser, 2015).

\section{Morphological Complexity}

Arabic language is one of the morphologically rich languages that has significant challenges to NLP systems in general (Abdul-Mageed et al., 2011). Arabic is a highly inflectional and derivational language and various forms can exist for the same Arabic word using different suffixes, affixes and prefixes (Shoukry and Rafea, 2012). Inflectional morphology refers to the process of adding extensions to a word while the Part Of Speech (POS) and the meaning of the word remain intact. For example, one word may have more than one lexical category in different contexts (El-Halees, 2011), such as a tense-based affix used as the present-tense prefix بـ/y-/ in - بـ/ynzr/,'he looks' (Al-Sabbagh and Girju, 2012). Derivational morphology refers to extracting new words from other words with modifying the core meaning of the word, e.g. the Arabic verb 'قال', /qạl/, 'to say', is the source for the Arabic noun 'قائل', /qayyl/, 'the person who is saying' and the noun 'قول', /qwl/, 'say (n)' (Habash, 2010). In addition, Arabic nouns and verbs are typically derived from a set of 10,000 roots (Mourad and Darwish, 2013) for different words and completely different meanings can be composed form the same root (Shoukry and Rafea, 2012). Almuqren and Cristea (2016) reported challenges of dealing with script of the Arabic language such as diacritization, negation and spelling errors. These different challenges in the Arabic language led to the lack of SA resources such as comprehensive sentiment lexica and corpora.

General tools for NPL are also moderately developed for Arabic. Khoja and Garside (1999) developed an Arabic stemmer. The author also developed a POS tagger for Arabic (Khoja, 2001). ISRI Arabic stemmer algorithm (Taghva et al., 2005) was implemented without a root-dictionary like Khoja Arabic Stemmer. The authors in (Pasha et al., 2014) developed MADAMIRA; a tool for morphological analysis and disambiguation. The authors in (Elfardy et al., 2014) introduced AIDA: Identifying Code Switching in Informal Arabic Text relying on Language Models and MADAMIRA (Pasha et al., 2014) to identify the class of each word in a given sentence.

\section{Dialectal Arabic}

Dealing with DA creates additional challenges (Zaidan and Callison-Burch, 2011; Refaee and Rieser, 2014). Using the different dialects in social media, where Arab users freely express themselves, adds more challenging to SA because the majority of the NLP tools for the Arabic language have been developed for MSA (alOwisheq et al., 2016). According to (Habash, 2010), Arabic dialects significantly differ from MSA in terms of phonology, morphology, lexical choice and syntax. The Arabic dialects are divided as:

- EA: Egyptian Arabic for Egypt and Sudan

- LA: Levantine Arabic for Lebanon, Syria, Palestine and Jordan

- GA: Gulf Arabic for Gulf area

- MA: Maghrebi Arabic for Morocco, Algeria, Tunisia, Mauritania and Libya 


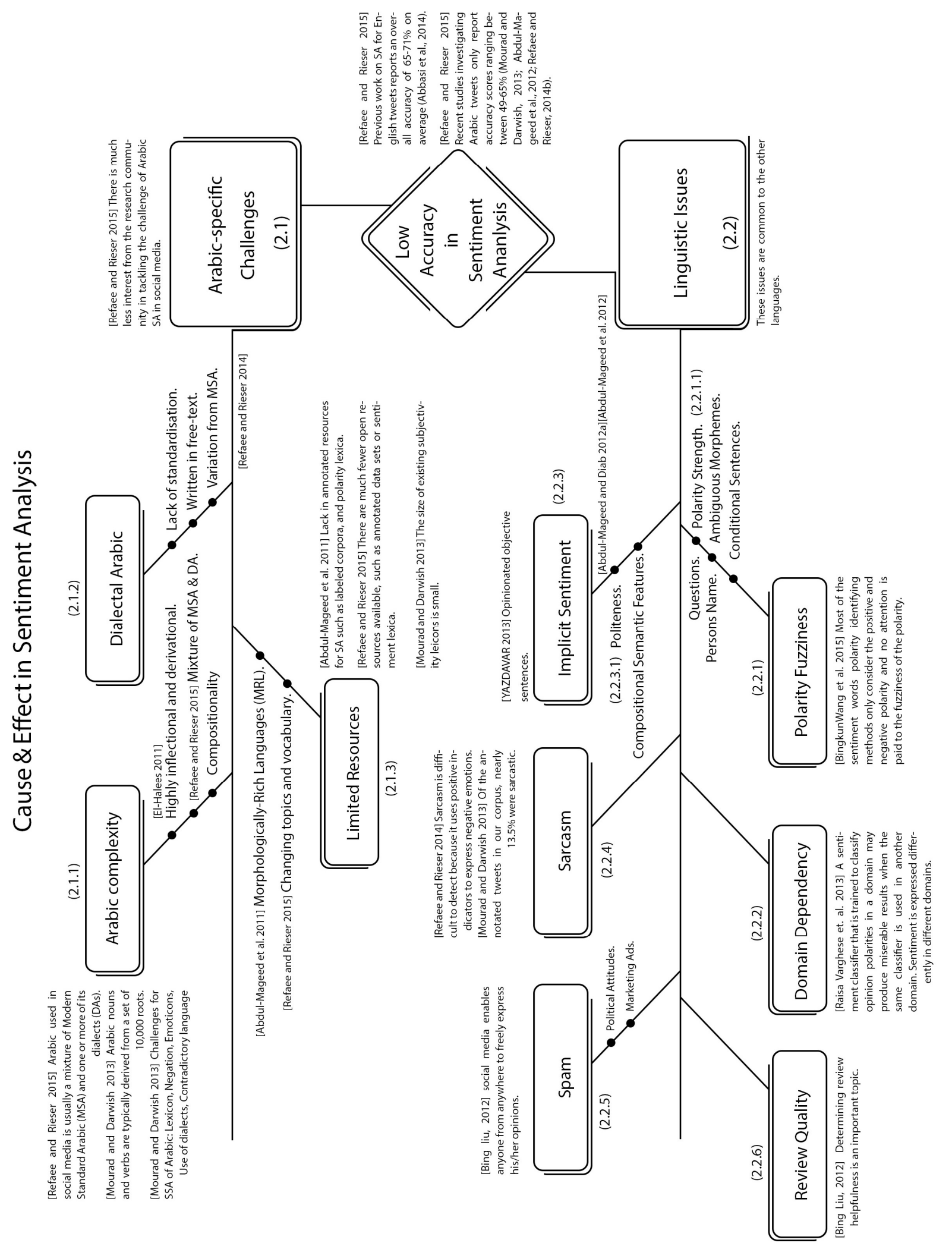

Fig. 1. Arabic Sentiment Analysis Challenging Issues 
Table 1. Arabic words and phrases with different meanings in Dialectal Arabic

\begin{tabular}{|c|c|c|c|c|c|c|c|}
\hline Word/phrase & MSA & EA & LA & GA & MA & IA & YA \\
\hline 'مبسوط/mbswț/“Happy-Rich’' & POS & POS & POS & POS & POS & NEG 'Struck' & POS \\
\hline 'الصلح اله حالك & POS & POS & NEG 'Bad phrase' & POS & POS & POS & POS \\
\hline lغاوي/ghạwy/‘Debauched’ & NEG & NEG & NEG & POS Beautiful & NEG & NEG & NEG \\
\hline l'أجنبي & NEU & NEU & POS 'VIP' & NEG 'Culturally opposite' & NEU & NEU & NEU \\
\hline
\end{tabular}

Habash (2010) added that each of the dialects contains three sub-dialects: City, Rural and Bedouin. These dialects lack standardization, written in free-text and vary from MSA. For example, the word 'العافية'; /ạl 'ạfÿ̈/; 'wellness' in MSA, but in the MA dialect, it means 'hell' and is widely used in a sentiment sentence like 'اله يعطيك العافية'; /ạllhy'tykạl 'ạfyẗ/; 'May Allah bless you' in almost all of the other Arabic dialects; however, in MA it means 'go to hell'. Also, the word 'مبسوط'; /mbswt!; ' happy' or 'rich', but in the IA it means 'severe beatings'. Table 1 compares the polarity in MSA words/phrases and DA.

\section{Limited Resources}

There is little focus from researchers on tackling the challenge of Arabic SA (Refaee and Rieser, 2014; El-Beltagy and Ali, 2013). Therefore, Arabic resources for SA are difficult to find. There is a lack of labeled corpora and polarity lexica (Refaee and Rieser, 2015; Abdul-Mageed et al., 2011; alOwisheq et al., 2016). In addition, the size of existing subjectivity lexicons is small (Mourad and Darwish, 2013). The complexity of the Arabic language as discussed earlier, affected negatively on the amount of existing resources.

Articles in the literature introduced Arabic corpora that are annotated for SA include:

- OCA Opinion Corpus for Arabic (Rushdi-Saleh et al., 2011) is an Arabic dataset consisting of 500 movie reviews

- COLABA (Diab et al., 2010) targeted EA, IA, LA and a much smaller effort on MA, ASTD (Nabil et al., 2015) contains $10 \mathrm{~K}$ tweets for the Egyptian Dialect

- YADAC (Al-Sabbagh and Girju, 2012) presented as a multi-genre dialectal Arabic corpus, using data from micro-blogs like twitter, blogs, forums and online market services

- AWATIF (Abdul-Mageed and Diab, 2012) is a multi-genre corpus of Modern Standard Arabic (MSA) contains 2855 news, 5342 Wikipedia talk pages and 2532 web forums conversations

- ElSahar and El-Beltagy (2015) built a multi-domain datasets consist of $33 \mathrm{~K}$ reviews for movies, hotels, restaurants and products

- Cotterell and Callison-Burch (2014) presented Multi-Dialect, Multi-Genre Corpus includes coverage of five dialects of Arabic: Egyptian, Gulf, Levantine, Maghrebi and Iraqi
- Refaee and Rieser (2014) claimed that their corpus would be the first Arabic SSA corpus, which is publicly released via the ELRA repository, they collected a corpus of 8,868 tweets (The corpus comprises a development set (7,503 tweets) and a test set $(1,365$ tweets $))$

In spite of these different resources, the variety of the Arabic language dialects still need more efforts to be covered. However, some domains like news have been covered in many resources, there are many other domains have not been targeted yet.

On the other hand, there are some polarity lexica such as the lexicon introduced in (Abdul-Mageed et al., 2011) that consisted of adjectives, the lexicon in (Mourad and Darwish, 2013) that used Machine Translation (MT) to translate an existing English lexicon and random graph walk to expand a manually prepared Arabic lexicon and ArSenL (Badaro et al., 2014b) that used existing resources including English SentiWordNet (ESWN) and Arabic WordNet to produce a large scale Standard Arabic sentiment lexicon. Depending on adjectives polarity lexicon is not enough, because of the richness of Arabic in expressing the feelings and sentiment. In addition, the change in polarity classification for each polarity word in different dialects, contexts and domains still an open issue (Liu, 2012; Varghese and Jayasree, 2013; Refaee and Rieser, 2014).

\section{General Linguistic Issues}

This section discusses the general linguistic issues that cause the low accuracy of SA in any language. We discuss these issues in relation to the Arabic language.

\section{Polarity Fuzziness}

Most of the methods used in sentiment classification considered the polarity (e.g., positivity and negativity) and do not pay attention to the polarity fuzziness (Wang et al., 2015). Sometimes it is hard to identify the polarity of a text. Even two humans may not agree on the same annotation; each can have a different point of view. A sentence containing sentiment words may not express any sentiment, such as questions, e.g., ' له استخدام الضوء هل use of the car main beam a violation?'. This question contains the word 'مخالف'; /mkhạlf/; 'violation' which bears a negative sentiment, but in this context, it bears no sentiment. Another example is conditional sentences,

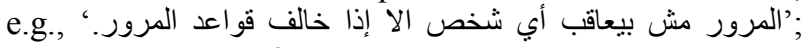

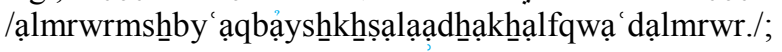


'Traffic police doesn't punish anyone but those who violate the traffic rules', which contains 'خالف'; / khallf/; 'violate', bearing negative sentiment but there is no sentiment in this conditional sentence (Liu, 2012). However, questions and conditional sentences may express sentiments, e.g. ' مدكن لو سمحت أعرف تأتبيرني هتخلص

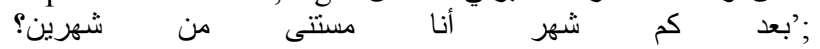
/mmknlwsmḥtạ r rftạ̉shyrtyhtkhِhṣb d km shhhrạnạmstny mnshhryn?/; 'Please let me know, how many months still for my visa to be issued, I have been waiting for two months?' and 'لو السائقين ملنزمين كانت خفت الزحمة.; /lwạlsạỷqynmltzmynkạntkḩftạlzḥmï./; 'If the drivers were committed, jams would be reduced'. The authors in (Jindal and Liu, 2006) proposed a novel rule mining and machine learning approach to identifying comparative sentences, which are useful in many applications such as marketing intelligence, product benchmarking and ecommerce. The authors in (Narayanan et al., 2009) carried out their study from both the linguistic and computational perspectives. The linguistic study focused on canonical tense patterns, which have proven useful in classification, while in the computational study, they automatically predicted whether opinions on topics were positive, negative, or neutral by building SVM models. In the Arabic language, using adjectives and nouns for people's names is common (Table 2). Thus, it is confusing to use one of them in a context similar to this: 'المجرم حسن جميل'; /ạlmjrmhsnjmyl./; which literally has two different meanings. (1) To use as adjectives, the phrase means 'The offender is good and beautiful'. (2) To use as a person's name, the phrase means 'Hassan Jamil is the offender'.

\section{Polarity Strength}

The sentiment word or phrase is a dominating factor in SA and the strength of the polarity is an important reference to the person's opinion or sentiment. To calculate the document-level sentiment scores, Taboada et al. (2011) used polarity dictionaries of sentiment words and phrases categorized by polarity and strength and employed with negations and intensifiers. To classify the sentiment strength in English text, Thelwall et al. (2011; 2012) proposed and improved a new algorithm, SentiStrength, using methods to exploit the de-facto grammar and spelling styles of cyberspace. Oraby et al. (2013) proposed a rule-based approach to extract the opinion-phrase using a sentiment lexicon with opinion indicators and after measuring the strength of the opinion, they developed the calculation method with four polarity categories (positive weak, positive strong, negative weak and negative strong). Arabic's strength level can be expressed in various forms. For instance, the

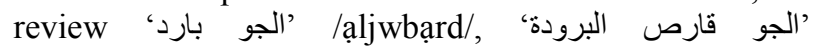

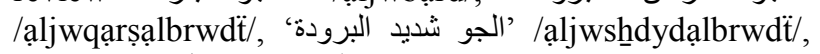

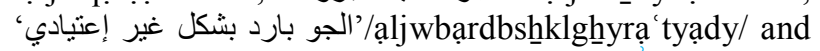
other forms used to say, 'The weather is cold, in different strength level of the coldness'.
Table 2. Adjectives and nouns used for people's names in Arabic

\begin{tabular}{|c|c|c|c|}
\hline Arabic & POS & Transliteration & English \\
\hline حسن & Adjective & /ḥsn/ & Good \\
\hline جمبل & Adjective & /jmyl/ & Beautiful \\
\hline جمال & Noun & /jmạl/ & Beauty \\
\hline كريم & Adjective & /krym/ & Generous \\
\hline 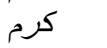 & Noun & /krm/ & Generosity \\
\hline سعبا & Adjective & /s'yd/ & Happy \\
\hline سعادة & Nouns & 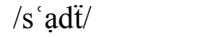 & Happiness \\
\hline
\end{tabular}

\section{Domain Dependency}

Sentiment is expressed differently in different domains (Varghese and Jayasree, 2013), so a sentiment classifier trained to classify opinion polarities in a domain may produce poor or useless results when used in another domain; the results are only accurate in the domain for which they are trained (Oraby et al., 2013). In addition, the sentiment word may have opposite orientations in different domains. For example, 'أقل';/ạq1/usually expresses negative sentiment, e.g., 'فلوس أقل من سعرها'، الفها'; /flwsạalmns 'rhạ/; 'Money is less than its worth', but it may also express a positive, e.g., ' الإجر اءات خلصت في وقت أقل /ạlạjậạtkhlṣtfywqtạql'/; 'The procedures were done in less time'. Another exampleis the sentence 'يستخدمون الهواتف'; /ystkhdmwnạlhwạtf/; 'They are using the phones'. This sentence in the domain of public services providing booking of appointment through the phones is positive, but when it is used in the context of people driving habits it bears a negative sentiment. Aue and Gamon (2005) discussed the challenges in using sentiment classifiers in new domains, showing that although the approaches are different, they all need a relatively labeled training dataset. Blitzer (2007) reported that domain adaptation addresses the situation in which labeled data from a source domain is used to train a model, but little or no labeled data from a target domain where the model will be applied. They applied learning representations, which minimize the difference between source and target domains. The proposed approach in (Wu et al., 2009) integrated the sentiment orientations of documents into the graph-ranking algorithm, which uses the accurate labels of old-domain documents as well as the 'pseudo' labels of new-domain documents. Pan et al. (2010) proposed a general framework for cross-domain sentiment classification. They first build a bipartite graph between domain-independent and domain-specific features and then they propose a Spectral Feature Alignment (SFA) algorithm to align the domain-specific words from the source and target domains into meaningful clusters with the help of domain-independent words as a bridge. ElSahar and El-Beltagy (2015) introduced large, multi-domain datasets for SA in the domains of movies, hotels, restaurants and products. Additionally, a multi-domain lexicon of 2,000 entries was extracted from the datasets. The researchers used SVM and K-Nearest (KNN) classifiers. SVM results 
were better than $\mathrm{KNN}$ ones and the best performing feature representations were the combination of the lexicon-based features with the other features.

\section{Implicit Sentiment}

Sentences with implicit sentiment are opinionated objective sentences (Yazdavar, 2013; Pang and Lee, 2004). Many sentences without sentiment words can also imply opinions. For example: 'This washer uses a lot of water' implies negative sentiment, 'After sleeping on the mattress for two days, a valley has formed in the middle' expresses a negative opinion, 'Phone doesn't fit pocket'implies that the phone size is inappropriate and 'Phone is cheap' implies a bad quality of the phone rather than a good price for it. In the Arabic language, implicit sentiment is popular in sentences like ' حسبي الله ونعم الوكيل'; /ḥsbyạllhwn 'mạlwkyl/; 'in Allah (God) I trust and $\mathrm{He}$ is best to trust', which is used when someone is beingoppressed. Implicit sentiment is also found in ' ده راجل طيب '; /dh rạjltyb/; 'he is a good man', as it may bear a negative sentiment when describing the man as unintelligent. Zhang and Liu (2011) studied the problem of objective nouns and sentences with implied opinions. They proposed a method that determines the feature polarity of opinion words that modify features and their surrounding context. Van de Kauter et al. (2015) introduced a fine-grained scheme for the annotation of polar sentiment, explicit sentiment (polar expressions) and implicit expressions of sentiment (polar facts) in text.

\section{Politeness and Euphemism}

Politeness is the practical application of good manners or etiquette. It is a culturally defined phenomenon and therefore what is considered polite in one culture can sometimes be quite rude or simply eccentric in another cultural context (Wikipedia, 2015). On the one hand, politeness may affect how people express their opinions or sentiments. For example, when we ask someone, 'Could you please activate my account'? The idea of blocking the account gives us a negative sentiment, but, in such a polite sentence, it is very hard to classify. On the other hand, direct requests like, 'Activate my account', have a negative sentiment (Abdul-Mageed and Diab, 2012). As mentioned above, politeness is changing according to the society and culture, so the different Arabic dialects present a big

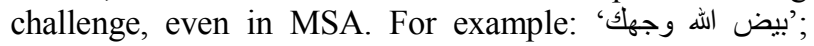
/bydạllhwjhk/; 'God whiten your face', is used in daily communication as a positive and polite sentence, while the original meaning for it is 'to wish death for someone who is bad'. Euphemism (e.g., use 'story' or 'cover' instead of 'lie') is also used widely in Arabic. For example, in the

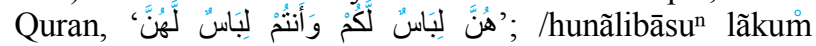
wāảntumālibāsun lãhunã/ [Al-Baqara 187] describes the relationship between husbands and wives, as the wives cover their husbands and protects them from sins.

\section{Sarcasm}

Sarcasm is difficult to detect (Refaee and Rieser, 2014) because it uses positive indicators to express negative emotions, e.g., 'What a great car! It stopped working in two days'. Sarcasm is not used in consumer reviews, but is very common in political reviews. Mourad and Darwish (2013) reported that in the annotated tweets in their corpus, nearly $13.5 \%$ were sarcastic. Using a positive sentiment in bad situations for the purpose of sarcasm is popular in Arabic. For instance, ' لقد أعطى الراتب لزوجته و هو 'يسنت، منافق ; /qdạ tỵ ạlrạtblzwjthwhwybstm, mnạfq/; 'He was happy when he gave the salary to his wife, he is hypocrite'. Davidov et al. (2010) used a semi-supervised approach to classify sentences in online product reviews into sarcastic classes. González-Ibánez et al. (2011) studied the problem of automatically detecting sarcasm in Twitter messages. Using an annotated corpus, they explored the contribution of linguistic and pragmatic features of tweets to the automatic identification of sarcastic messages and found that the three pragmatic features-ToUser, smiley and frown-were among the ten most discriminating features in the classification tasks. Maynard and Greenwood (2014) investigated the Twitter sarcasm characteristics and the effect of sarcasm on sentiment analysis.

\section{Spam}

The abundance of social media allows spammers to post fake opinions to promote a product or to discredit another. Spam is also spread in political and governmental reviews. Jindal and Liu (2007) studied the spam review problem in a manufactured products dataset and a logistic regression was performed. Three types of duplicate reviews are most likely to be spam: (1) From different userids on the same product, (2) from the same userid on different products and (3) from different userids on different products. Jindal and Liu (2008) reported three types of spam reviews: (1) Fake reviews: Untruthful reviews containing positive or negative opinions about target entities (products or services) in order to promote or damage their reputations, (2) brand reviews: Do not comment on the specific products or services but on the brands or the manufacturers and (3) non-reviews. There are two main subtypes: Advertisements and other irrelevant texts containing no opinions (e.g., questions, answers and random texts). Strictly speaking, these are not opinion spam, as they do not give user opinions.

\section{Review Quality}

The quality, usefulness, helpfulness, or the utility of the review is important to be taken into account in SA. A review may not be actually spam, but neither is it helpful. For example, a review targeting a brand like Apple, while evaluating a product such as IPhone 7, may be Apple is a good brand but the evaluated product is not good. Also, greeting comments such as ' صباح الخير 
وو النور. /ssbạhạlkhyrwạlnwr/; 'good morning'. Consider this comment on an organization's Facebook post. It appears to bear a positive sentiment, while it is not helpful in evaluating the sentiment toward the organization. As using greetings may be followed by a question or a complaint against the introduced services. Kim et al. (2006) proposed an algorithm for automatically assessing helpfulness and ranking reviews according to helpfulness using an SVM regression system. Ghose and Ipeirotis (2007) find reviews that include a mixture of subjective and objective elements that are considered more informative or helpful by the users.

\section{Conclusion}

Sentiment analysis have been used in various applications in public and customer opinion studies such as social, news and commerce domains. The accuracy of the analysis has a direct effect upon the decision-making capacity of businesses and governments. Therefore, the need for efficient sentiment analysis systems is on the rise. Even though the popularity of using Arabic language in the internet is on the rise, there are limited efforts in Arabic sentiment analysis and building of necessary resources, namely lexica and corpora. The work on building Arabic polarity lexicon often relies on the English available lexicons which may be affected by the different cultures. Also, composing the lexicon from adjectives and neglecting the nouns and other POSs is not enough. Most Arabic corpora are unpublished and the available ones still need more efforts to cover the multi-dialects and the different domains issue. There are few attempts to use Fuzzy logic to raise the accuracy of Sentiment Analysis. In spite of the mentioned efforts, the sentiment analysis in Arabic language still has many unsolved issues. Table 3 below shows some of the related works to highlight the different Approaches and methods which used to address the dimensions of sentiment analysis low accuracy problem. This includes Sources/Genres, Domains, Dialects and Linguistic Issues. Also the table shows how they dealt with the Arabic-specific challenges and illustrates their outputs either annotated corpus or sentiment lexicon. From the table, it is clear that dealing with the sentiment analysis low accuracy requires to take into account different dimensions that affect the polarity strength and direction. Domain dependency still considered an open issue because the previous woks aedomain specific.

Table 3. Related works

\begin{tabular}{|c|c|c|c|c|c|c|c|c|}
\hline \multicolumn{9}{|c|}{ Dimensions of SA Low Accuracy Problem } \\
\hline & $\begin{array}{l}\text { Approaches/ } \\
\text { Methods }\end{array}$ & $\begin{array}{l}\text { Sources/ } \\
\text { Genres }\end{array}$ & Domains & Dialects & $\begin{array}{l}\text { Linguistic } \\
\text { Issues }\end{array}$ & $\begin{array}{l}\text { Arabic- } \\
\text { specific } \\
\text { challenges }\end{array}$ & Output & Notes \\
\hline $\begin{array}{l}\text { Badaro et al. } \\
\text { (2014a) }\end{array}$ & $\begin{array}{l}\text { Building Lexicon } \\
\text { using Arabic } \\
\text { WordNet-based } \\
\text { Approach, } \\
\text { (Mapping AWN to } \\
\text { ESWN and } \\
\text { Mapping SAMA to } \\
\text { AWN) and English } \\
\text { Gloss-based } \\
\text { Approach }\end{array}$ & $\begin{array}{l}\text { ESWN, Arabic } \\
\text { WordNet and } \\
\text { the Standard } \\
\text { Arabic } \\
\text { Morphological } \\
\text { Analyzer (SAMA). }\end{array}$ & General & MSA & - & $\begin{array}{l}\text { Limited } \\
\text { resources }\end{array}$ & $\begin{array}{l}\text { Arabic } \\
\text { SALexicon }\end{array}$ & $\begin{array}{l}\text { Used the existed } \\
\text { English resources } \\
\text { and Machine } \\
\text { Translation, only } \\
\text { for MSA. }\end{array}$ \\
\hline $\begin{array}{l}\text { Abdul-Mageed } \\
\text { and Diab } \\
\text { (2014) }\end{array}$ & $\begin{array}{l}\text { Building resources: } \\
\text { Manual, Statistic } \\
\text { (PMI, Popular word } \\
\text { association measure } \\
\text { and MT). }\end{array}$ & $\begin{array}{l}\text { Newswire, chat, } \\
\text { tweets, YouTube } \\
\text { comments }\end{array}$ & Genera, news & $\begin{array}{l}\text { MSA, EA, } \\
\text { LA }\end{array}$ & $\begin{array}{l}\text { Dialectal } \\
\text { Arabic }\end{array}$ & $\begin{array}{l}\text { Limited } \\
\text { resources }\end{array}$ & $\begin{array}{l}\text { Multi-genre, } \\
\text { multi DAmulti- } \\
\text { lingual Lexicon }\end{array}$ & $\begin{array}{l}\text { Limited specific } \\
\text { domains and } \\
\text { dialects. }\end{array}$ \\
\hline $\begin{array}{l}\text { Abdul-Mageed } \\
\text { and Diab } \\
(2012)\end{array}$ & $\begin{array}{l}\text { Building resources: } \\
\text { Manual (TWO } \\
\text { ANNTATORS), } \\
\text { (387 turkers) }\end{array}$ & $\begin{array}{l}\text { Penn Arabic } \\
\text { Treebank (PATB), } \\
\text { Wikipedia Talk } \\
\text { Pages, Web } \\
\text { Forums }\end{array}$ & $\begin{array}{l}\text { News (e.g., } \\
\text { political, } \\
\text { economic, } \\
\text { sports), } \\
\text { Political. }\end{array}$ & MSA & $\begin{array}{l}\text { Implicit } \\
\text { (Politeness), } \\
\text { Perspective, } \\
\text { Illocutionary } \\
\text { speech. }\end{array}$ & $\begin{array}{l}\text { Limited } \\
\text { resources }\end{array}$ & $\begin{array}{l}\text { Multi-Genre } \\
\text { Corpus }\end{array}$ & $\begin{array}{l}\text { For news and } \\
\text { political and } \\
\text { target the MSA } \\
\text { only. }\end{array}$ \\
\hline $\begin{array}{l}\text { Rushdi- } \\
\text { Saleh et al. } \\
\text { (2011) }\end{array}$ & $\begin{array}{l}\text { Corpus-Based, } \\
\text { SVM and NB }\end{array}$ & $\begin{array}{l}\text { Movies webpages } \\
\text { and blogs }\end{array}$ & Movies & $\begin{array}{l}\text { Mixed with } \\
\text { verification } \\
\text { of clarity. }\end{array}$ & - & $\begin{array}{l}\text { Limited } \\
\text { resources }\end{array}$ & $\begin{array}{l}\text { Arabic } \\
\text { SACorpus }\end{array}$ & $\begin{array}{l}\text { Specific for } \\
\text { movie domain, } \\
\text { based on blog } \\
\text { reviews. }\end{array}$ \\
\hline $\begin{array}{l}\text { Refaee and } \\
\text { Rieser } \\
(2014)\end{array}$ & $\begin{array}{l}\text { Corpus-Based, } \\
\text { Semi-supervised } \\
\text { online learning, } \\
\text { Inter-annotator } \\
\text { agreement } \\
\text { (Cohen's Kappa) }\end{array}$ & Twitter & General & Mixed. & $\begin{array}{l}\text { Arabic complexity, } \\
\text { Dialectal Arabic, } \\
\text { Mixed sentiments, } \\
\text { Sarcasm, Fuzziness, } \\
\text { negation. }\end{array}$ & $\begin{array}{l}\text { Limited } \\
\text { resources }\end{array}$ & $\begin{array}{l}\text { Multi DA } \\
\text { Twitter corpus, } \\
\text { words and phrases } \\
\text { annotated lexicon. }\end{array}$ & $\begin{array}{l}\text { Limited size, } \\
\text { based on Twitter } \\
\text { only, using } \\
\text { MADA+TOKEN } \\
\text { which is MSA } \\
\text { on DA. }\end{array}$ \\
\hline $\begin{array}{l}\text { Al-Sabbagh } \\
\text { and Girju } \\
(2012)\end{array}$ & Corpus-Based & $\begin{array}{l}\text { Twitter, Blog/ } \\
\text { Forums, Online } \\
\text { market services. }\end{array}$ & $\begin{array}{l}\text { General, } \\
\text { Market } \\
\text { services. }\end{array}$ & $\begin{array}{l}\text { Egyptian } \\
\text { Arabic (EA) }\end{array}$ & Dialectal Arabic & $\begin{array}{l}\text { Limited } \\
\text { resources }\end{array}$ & $\begin{array}{l}\text { Multi-genre } \\
\text { DA corpus }\end{array}$ & $\begin{array}{l}\text { Specific for } \\
\text { Egyptian Arabic. }\end{array}$ \\
\hline $\begin{array}{l}\text { ElSahar and } \\
\text { El-Beltagy } \\
(2015)\end{array}$ & $\begin{array}{l}\text { Corpus-Based } \\
\text { and Lexicon-Based }\end{array}$ & $\begin{array}{l}\text { Reviewing websites: } \\
\text { Tripadvisor, Qaym, } \\
\text { elcinma.com and } \\
\text { souq.com }\end{array}$ & $\begin{array}{l}\text { Movies, hotels, } \\
\text { restaurants and } \\
\text { products }\end{array}$ & & $\begin{array}{l}\text { Domain } \\
\text { dependency }\end{array}$ & $\begin{array}{l}\text { Limited } \\
\text { resources }\end{array}$ & $\begin{array}{l}\text { Multi-domain } \\
\text { corpus, } \\
\text { multi-domain } \\
\text { lexicons }\end{array}$ & $\begin{array}{l}\text { For specific } \\
\text { domains. }\end{array}$ \\
\hline
\end{tabular}


Moreover, dialectal Arabic still not covered and needs to be addressed especially with different domains. In this study, the issues which cause the sentiment analysis low accuracy problem are discussed based on two main components: Arabic-specific challenges and general linguistic issues. The Arabic-specific challenges divided into three main parts: Morphological complexity, limited resources and dialects. The general linguistic issues include polarity fuzziness, implicit sentiment, sarcasm, polarity strength, spam, review quality issues and domain dependence.

\section{Acknowledgment}

This work was made possible by NPRP 6-716-1-138 grant from Qatar National Research Fund (a member of Qatar Foundation). The statements made herein are solely the responsibility of the authors.

\section{Author's Contributions}

All authors equally contributed in this work.

\section{Ethics}

This article is original and contains unpublished material. The corresponding author confirms that all of the other authors have read and approved the manuscript and no ethical issues involved.

\section{References}

Abdul-Mageed, M. and M.T. Diab, 2012. AWATIF: A multi-genre corpus for modern standard arabic subjectivity and sentiment analysis. LREC.

Abdul-Mageed, M. and M.T. Diab, 2014. SANA: A large scale multi-genre, multi-dialect lexicon for Arabic subjectivity and sentiment analysis. Proceedings of the 9th International Conference on Language Resources and Evaluation, May 26-31, European Language Resources Association, Reykjavik, Iceland, pp: 1162-69.

Abdul-Mageed, M., M.T. Diab and M. Korayem, 2011. Subjectivity and sentiment analysis of modern standard Arabic. Proceedings of the 49th Annual Meeting of the Association for Computational Linguistics: Human Language Technologies, Jun. 19-24, Association for Computational Linguistics, USA., pp: 587-591.

Ahire, S., 2014. A survey of sentiment lexicons.

Alhumoud, S.O., M.I. Altuwaijri, T.M. Albuhairi and W.M. Alohaideb, 2015. Survey on Arabic sentiment analysis in twitter. Int. Sci. 9: 364-368.

Al-Kabi, M.N, I.M. Alsmadi, A.H. Gigieh, H.A. Wahsheh and M.M. Haidar, 2014. Opinion mining and analysis for Arabic language. Int. J. Adv. Comput. Sci. Applic., 5: 181-95.

DOI: 10.14569/IJACSA.2014.050528
Al-Kabi, M.N., N.A. Abdulla and M. Al-Ayyoub, 2013. An analytical study of Arabic sentiments: Maktoob case study. Proceedings of the 8th International Conference for Internet Technology and Secured Transactions, Dec. 9-12, IEEE Xplore Press, pp: 89-94. DOI: 10.1109/ICITST.2013.6750168

Almuqren, L. and A.I. Cristea, 2016. Framework for sentiment analysis of Arabic text. Proceedings of the 27th ACM Conference on Hypertext and Social Media, Jul. 10-13, ACM, Nova Scotia, Canada, pp: 315-317. DOI: $10.1145 / 2914586.2914610$

alOwisheq, A., S. alHumoud, N. alTwairesh and T. alBuhairi, 2016. Arabic sentiment analysis resources: A survey. 8th International Conference on Social Computing and Social Media, Jul. 17-22, Springer, Canada, pp: 267-278.

DOI: 10.1007/978-3-319-39910-2 25

Al-Sabbagh, R. and R. Girju, 2012. YADAC: Yet another dialectal Arabic corpus. Proceedings of the 8th International Conference on Language Resources and Evaluation, May 23-25, European Language Resources Association, Istanbul, Turkey, pp: 2882-89.

Al Sallab, A.A., H.M. Hajj, R. Baly and K. Shaban, 2015. Deep learning models for sentiment analysis in Arabic. Proceedings of the 2nd Workshop on Arabic Natural Language Processing, Jul. 26-31, Association for Computational Linguistics, Beijing, China, pp: 9-17.

Al-Sughaiyer, I.A. and I.A. Al-Kharashi, 2004. Arabic morphological analysis techniques: A comprehensive survey. J. Am. Society Inform. Sci. Technol., 55: 189-213. DOI: 10.1002/asi.10368

Assiri, A., A. Emam and H. Aldossari, 2015. Arabic sentiment analysis: A survey. Int. J. Adv. Comput. Sci. Applic., 1: 75-85.

DOI: 10.14569/IJACSA.2015.061211

Aue, A. and M. Gamon, 2005. Customizing sentiment classifiers to new domains: A case study. Proceedings of the 5th International Conference on Recent Advances in Natural Language Processing, (NLP' 05).

Badaro, G., R. Baly, H. Hajj, N. Habash and W. El-Hajj, 2014a. A large scale Arabic sentiment lexicon for Arabic opinion mining. Proceedings of the Workshop on Arabic Natural Langauge Processing, Oct. 25-25, Association for Computational Linguistics, Doha, Qatar, pp: 165-173.

Badaro, G., R. Baly, H. Hajj, N. Habash and W. El-hajj et al., 2014b. An efficient model for sentiment classification of Arabic tweets on mobiles. Proceedings of the Qatar Foundation Annual Research Conference, Nov. 13-13, Doha, Qatar.

Blitzer, J., 2007. Domain adaptation of natural language processing systems. University of Pennsylvania.

Bollen, J., H. Mao and X. Zeng, 2011. Twitter mood predicts the stock market. J. Comput. Sci., 2: 1-8. DOI: $10.1016 /$ j.jocs.2010.12.007 
Buche, A., D. Chandak and A. Zadgaonkar, 2013. Opinion mining and analysis: A survey. arXiv preprint arXiv:1307.3336.

Cotterell, R. and C. Callison-Burch, 2014. A multidialect, multi-genre corpus of informal written Arabic. Proceedings of the Ninth International Conference on Language Resources and Evaluation, May 26-31, European Language Resources Association, Reykjavik, Iceland.

Davidov, D., O. Tsur and A. Rappoport, 2010. Semisupervised recognition of sarcastic sentences in twitter and Amazon. Proceedings of the 14th Conference on Computational Natural Language Learning, Jul. 15-16, Association for Computational Linguistics, Uppsala, Sweden, pp: 107-16.

Diab, M., N. Habash, O. Rambow, M. Altantawy and Y. Benajiba, 2010. COLABA: Arabic dialect annotation and processing. Proceedings of the LREC Workshop on Semitic Language Processing, (SLP' 10), pp: 66-74.

El-Beltagy, S.R. and A. Ali, 2013. Open issues in the sentiment analysis of Arabic social media: A case study. 9th proceedings of the International Conference on Innovations in Information Technology, Mar. 17-19, IEEE Xplore Press, pp: 215-220. DOI: 10.1109/Innovations.2013.6544421

El-Halees, A., 2011. Arabic opinion mining using combined classification approach.

Elfardy, H., M. Al-Badrashiny and M. Diab, 2014. AIDA: Identifying code switching in informal Arabic text. Proceedings of the 1st Workshop on Computational Approaches to Code Switching, Oct. 25-25, Association for Computational Linguistics, Doha, Qatar, pp: 94-101.

ElSahar, H. and S.R. El-Beltagy, 2015. Building large Arabic multi-domain resources for sentiment analysis. Proceedings of the 16th International Conference on Computational Linguistics and Intelligent Text Processing, Apr. 14-20, Springer, Cairo, Egypt, pp: 23-34. DOI: 23-34. DOI: 10.1007/978-3-319-18117-2_2

Farra, N., E. Challita, R.A. Assi and H. Hajj, 2010. Sentence-level and document-level sentiment mining for Arabic texts. Proceedings of the IEEE International Conference on Data Mining Workshops, Dec. 13-13, IEEE Xplore Press, pp: 1114-1119. DOI: 10.1109/ICDMW.2010.95

Ghose, A. and P.G. Ipeirotis, 2007. Designing novel review ranking systems: Predicting the usefulness and impact of reviews. Proceedings of the 9th International Conference on Electronic Commerce, Aug. 19-22, ACM, Minneapolis, pp: 303-10.

DOI: $10.1145 / 1282100.1282158$
González-Ibánez, R., S. Muresan and N. Wacholder, 2011. Identifying sarcasm in Twitter: A closer look. Proceedings of the 49th Annual Meeting of the Association for Computational Linguistics: Human Language Technologies, Jun. 19-24, Association for Computational Linguistics, Portland, Oregon, pp: 581-86.

Habash, N.Y., 2010. Introduction to Arabic natural language processing. Synthesis Lect. Human Lang. Technol., 3: 1-187. DOI: 10.2200/S00277ED1V01Y201008HLT010

Jindal, N. and B. Liu, 2006. Identifying comparative sentences in text documents. Proceedings of the 29th Annual International ACM SIGIR Conference on Research and Development in Information Retrieval, Aug. 06-11, ACM, Seattle, Washington, pp: 244-51. DOI: 10.1145/1148170.1148215

Jindal, N. and B. Liu, 2007. Review spam detection. Proceedings of the 16th International Conference on World Wide Web, May 08-12, ACM, Banff, Alberta, pp: 1189-90. DOI: $10.1145 / 1242572.1242759$

Jindal, N. and B. Liu, 2008. Opinion spam and analysis. Proceedings of the International Conference on Web Search and Data Mining, Feb. 11 - 12, ACM, Palo Alto, California, pp: 219-30. DOI: $10.1145 / 1341531.1341560$

Kaur, A. and N. Duhan, 2015. A survey on opinion mining and sentiment analysis. Knowledge-Based Syst., 89: 14-46. DOI: 10.1016/j.knosys.2015.06.015

Khoja, S., 2001. APT: Arabic part-of-speech tagger. Proceedings of the Student Workshop at NAACL, (NAACL' 01), pp: 20-25.

Khoja, S. and R. Garside, 1999. Stemming Arabic text. Lancaster University, Lancaster, UK.

Kim, S.M., P. Pantel, T. Chklovski and M. Pennacchiotti, 2006. Automatically assessing review helpfulness. Proceedings of the Conference on Empirical Methods in Natural Language Processing, Jul. 22-23, Association for Computational Linguistics, Sydney, Australia, pp: 423-30.

Korayem, M., 2016. Sentiment/subjectivity analysis survey for languages other than English. arXiv preprint arXiv:1601.00087.

Korayem, M., D. Crandall and M. Abdul-Mageed, 2012. Subjectivity and sentiment analysis of Arabic: A survey. Proceedings of the 1st International Conference on Advanced Machine Learning Technologies and Applications, Dec. 8-10, Springer, Cairo, Egypt, pp: 128-39. DOI: 10.1007/978-3-642-35326-0_14

Kontopoulos, E., C. Berberidis, T. Dergiades and N. Bassiliades, 2013. Ontology-based sentiment analysis of twitter posts. Expert Syst. Applic., 40: 4065-4074. DOI: 10.1016/j.eswa.2013.01.001 
Li, Y.M. and T.Y. Li, 2013. Deriving market intelligence from microblogs. Decision Support Syst., 55: 206-217. DOI: 10.1016/j.dss.2013.01.023

Liu, B., 2012. Sentiment analysis and opinion mining. Synthesis Lect. Human Lang. Technol., 5: 1-167. DOI: 10.2200/S00416ED1V01Y201204HLT016

Liu, B. and L. Zhang, 2012. A Survey of Opinion Mining and Sentiment Analysis. In: Mining Text Data, Aggarwal, C.C. and C.X. Zhai (Eds.), Springer, pp: 415-63.

Mahadik, A. and A. Bharambe, 2015. Aspect based opinion mining and ranking: Survey. Int. J. Curr. Eng. Technol., 5: 3589-3592.

Maynard, D. and M.A. Greenwood, 2014. Who cares about sarcastic tweets? Investigating the impact of sarcasm on sentiment analysis. Proceedings of the LREC, (LREC' 14), pp: 4238-43.

Mourad, A. and K. Darwish, 2013. Subjectivity and sentiment analysis of modern standard Arabic and Arabic microblogs. Proceedings of the 4th Workshop on Computational Approaches to Subjectivity, Sentiment and Social Media Analysis, Jun. 14-14, Association for Computational Linguistics, Atlanta, Georgia, pp: 55-64.

Nabil, M., M. Aly and A.F. Atiya, 2015. ASTD: Arabic sentiment tweets dataset. Proceedings of the Conference on Empirical Methods in Natural Language Processing, Sept. 17-21, Association for Computational Linguistics, Lisbon, Portugal, pp: 2515-19.

Narayanan, R., B. Liu and A. Choudhary, 2009. Sentiment analysis of conditional sentences. Proceedings of the Conference on Empirical Methods in Natural Language Processing, Aug. 06-07, Association for Computational Linguistics, Singapore, pp: 180-89.

Negi, S. and P. Buitelaar, 2014. INSIGHT Galway: Syntactic and lexical features for aspect based sentiment analysis. Proceedings of the 8th International Workshop on Semantic Evaluation, Aug. 23-24, Dublin, Ireland, pp: 346-350.

Oraby, S., Y. El-Sonbaty and M.A. El-Nasr, 2013. Finding opinion strength using rule-based parsing for Arabic sentiment analysis. Proceedings of the 12th Mexican International Conference on Artificial Intelligence, Advances in Soft Computing and Its Applications, Nov. 24-30, Springer, Mexico, pp: 509-20. DOI: 10.1007/978-3-642-45111-9 44

Pan, S.J., X. Ni, J.T. Sun, Q. Yang and Z. Chen, 2010. Cross-domain sentiment classification via spectral feature alignment. Proceedings of the 19th International Conference on World Wide Web, Apr. 26-30, ACM, North Carolina, USA., pp: 751-60. DOI: $10.1145 / 1772690.1772767$
Pang, B. and L. Lee, 2004. A sentimental education: Sentiment analysis using subjectivity summarization based on minimum cuts. Proceedings of the 42nd Annual Meeting on Association for Computational Linguistics, Jul. 21-26, Association for Computational Linguistics, Barcelona, Spain. DOI: $10.3115 / 1218955.1218990$

Pang, B., L. Lee and S. Vaithyanathan, 2002. Thumbs up?: Sentiment classification using machine learning techniques. Proceedings of the Conference on Empirical Methods in Natural Language Processing, (NLP' 02), Association for Computational Linguistics, pp: 79-86. DOI: 10.3115/1118693.1118704

Pasha, A., M. Al-Badrashiny, M. Diab, A. El Kholy and R. Eskander et al., 2014. Madamira: A fast, comprehensive tool for morphological analysis and disambiguation of Arabic. Proceedings of the Language Resources and Evaluation Conference, May 26-31, European Language Resources Association, Reykjavik, Iceland.

Pontiki, M., D. Galanis, H. Papageogiou, S. Manandhar and I. Androutsopoulos, 2015. Semeval-2015 task 12: Aspect based sentiment analysis. Proceedings of the 9th International Workshop on Semantic Evaluation, Jun. 4-5, Association for Computational Linguistics, Denver, Colorado, pp: 486-495. DOI: $10.18653 / \mathrm{v} 1 / \mathrm{S} 15-2082$

Pontiki, M., H. Papageorgiou, D. Galanis, I. Androutsopoulos and J. Pavlopoulos et al., 2014. Semeval-2014 task 4: Aspect based sentiment analysis. Proceedings of the 8th International Workshop on Semantic Evaluation, Aug. 23-24, Dublin, Ireland, pp: 27-35. DOI: $10.3115 / \mathrm{v} 1 / \mathrm{S} 14-2004$

Baly, R., R. Hobeica, H. Hajj, W. El-hajj and K.B. Shaban et al., 2016. A meta-framework for modeling the human reading process in sentiment analysis. ACM Trans. Inform. Syst. DOI: $10.1145 / 2950050$

Refaee, E. and V. Rieser, 2014. An Arabic twitter corpus for subjectivity and sentiment analysis. Proceedings of the 9th International Conference on Language Resources and Evaluation, May 26-31, European Language Resources Association, Reykjavik, Iceland.

Refaee, E. and V. Rieser, 2015. Benchmarking machine translated sentiment analysis for Arabic tweets. Proceedings of NAACL-HLT 2015 Student Research Workshop, Jun. 1-1, Association for Computational Linguistics, Denver, Colorado, pp: 71-78.

Vinodhini, R.M. and G. Chandrasekaran, 2014. Performance evaluation of machine learning classifiers in sentiment mining. arXiv preprint arXiv:1402.3891.

Rushdi-Saleh, M., M.T. Martín-Valdivia, L.A. Ureña-López and J.M. Perea-Ortega, 2011. OCA: Opinion corpus for Arabic. J. Am. Society Inform. Sci. Technol., 62: 2045-54. DOI: 10.1002/asi.21598 
Sadegh, M., R. Ibrahim and Z.A. Othman, 2012. Opinion mining and sentiment analysis: A survey. Int. J. Comput. Technol., 2: 171-78.

Schouten, K. and F. Frasincar, 2015. Survey on aspectlevel sentiment analysis. IEEE Trans. Knowledge Data Eng., 28: 813-830.

DOI: $10.1109 /$ TKDE.2015.2485209

Sharma, A. and S. Dey, 2012. A document-level sentiment analysis approach using artificial neural network and sentiment lexicons. ACM SIGAPP Applied Comput. Rev., 12: 67-75.

Shoukry, A. and A. Rafea, 2012. Sentence-level Arabic sentiment analysis. Proceedings of the International Conference on Collaboration Technologies and Systems, May 21-25, IEEE Xplore Press, pp: 54650. DOI: $10.1109 /$ CTS.2012.6261103

Socher, R., A. Perelygin, J.Y. Wu, J. Chuang and C.D. Manning et al., 2013. Recursive deep models for semantic compositionality over a sentiment Treebank. Proceedings of the Conference on Empirical Methods in Natural Language Processing, (NLP' 13), pp: 1642-1642.

IWS, 2016. Internet World Stats.

Taboada, M., J. Brooke, M. Tofiloski, K. Voll and M. Stede, 2011. Lexicon-based methods for sentiment analysis. Comput. Linguist., 37: 267-307. DOI: 10.1162/COLI_a_00049

Taghva, K., R. Elkhoury and J.S. Coombs, 2005. Arabic stemming without a root dictionary. Proceedings of the International Conference on Information Technology: Coding and Computing, Apr. 4-6, IEEE Xplore Press, pp: 152-57. DOI: 10.1109/ITCC.2005.90

Tang, D., F. Wei, N. Yang, M. Zhou and T. Liu et al., 2014. Learning sentiment-specific word embedding for twitter sentiment classification. Proceedings of the 52nd Annual Meeting of the Association for Computational Linguistics, Jun. 23-25, Association for Computational Linguistics, Baltimore, Maryland, USA., pp: 1555-1565. 2014.

Tang, H., S. Tan and X. Cheng, 2009. A survey on sentiment detection of reviews. Expert Syst. Applic., 36: 10760-73. DOI: 10.1016/j.eswa.2009.02.063

Thelwall, M., K. Buckley and G. Paltoglou, 2012. Sentiment strength detection for the social web. J. Am. Society Inform. Sci. Technol., 63: 163-73. DOI: 10.1002 /asi.21662

Thelwall, M., K. Buckley, G. Paltoglou, D. Cai and A. Kappas, 2011. Sentiment strength detection in short informal text. J. Am. Society Inform. Sci. Technol., 61: 419-419.

Turney, P.D., 2002. Thumbs up or thumbs down?: Semantic orientation applied to unsupervised classification of reviews. Proceedings of the 40th Annual Meeting on Association for Computational Linguistics, Jul. 07-12, Association for Computational Linguistics, Pennsylvania, pp: $417-$ 24. DOI: $10.3115 / 1073083.1073153$
Van de Kauter, M., B. Desmet and V. Hoste, 2015. The good, the bad and the implicit: A comprehensive approach to annotating explicit and implicit sentiment. Lang. Resources Evaluat., 49: 685-720. DOI: $10.1007 / \mathrm{s} 10579-015-9297-4$

Varghese, R. and M. Jayasree, 2013. Aspect based sentiment analysis using support vector machine classifier. Proceedings of the International Conference on Advances in Computing, Communications and Informatics, Aug. 22-25, IEEE Xplore Press, pp: 158186. DOI: 10.1109/ICACCI.2013.6637416

Wang, B., Y. Huang, X. Wu and X. Li, 2015. A fuzzy computing model for identifying polarity of Chinese sentiment words. Comput. Intell. Neurosci., 2015: 525437-525449. DOI: 10.1155/2015/525437

Wiebe, J.M., R.F. Bruce and T.P. O'Hara, 1999. Development and use of a gold-standard data set for subjectivity classifications. Proceedings of the 37th Annual Meeting of the Association for Computational Linguistics on Computational Linguistics, Jun. 20-26, Association for Computational Linguistics, Maryland, pp: 246-53. DOI: 10.3115/1034678.1034721

Wiegand, M., A. Balahur, B. Roth, D. Klakow and A. Montoyo, 2010. A survey on the role of negation in sentiment analysis. Proceedings of the Workshop on Negation and Speculation in Natural Language Processing, Association for Computational Linguistics, pp: 60-68.

Wikipedia, 2015. 'wikipedia'.

Wu, Q., S. Tan and X. Cheng, 2009. Graph ranking for sentiment transfer. Proceedings of the ACL-IJCNLP Conference Short Papers, Aug. 04-04, Association for Computational Linguistics, Suntec, Singapore, pp: $317-20$.

Yadav, S.K., 2015. Sentiment analysis and classification: A survey. Int. J. Adv. Res. Comput. Sci. Manage. Stud., 3: 113-121.

Yazdavar, A.H., 2013. Fuzzy based implicit sentiment analysis on quantitative sentences. MSc Thesis, Universiti Teknologi Malaysia.

Yuan, Y. and Y. Zhou, 2015. Twitter Sentiment Analysis with Recursive Neural Networks.

Zaidan, O.F. and C. Callison-Burch, 2011. The Arabic online commentary dataset: An annotated dataset of informal Arabic with high dialectal content. Proceedings of the 49th Annual Meeting of the Association for Computational Linguistics: Human Language Technologies, Jun. 19-24, Association for Computational Linguistics, Oregon, pp: 37-41.

Zhang, L. and B. Liu, 2011. Identifying noun product features that imply opinions. Proceedings of the 49th Annual Meeting of the Association for Computational Linguistics: Human Language Technologies, Jun. 19-24, Association for Computational Linguistics, Portland, Oregon, pp: 575-80. 